\title{
Properties of Neon, Magnesium, and Silicon Primary Cosmic Rays Results from the Alpha Magnetic Spectrometer
}

M. Aguilar, ${ }^{30}$ L. Ali Cavasonza, ${ }^{1}$ G. Ambrosi,${ }^{36}$ L. Arruda, ${ }^{28}$ N. Attig, ${ }^{24}$ F. Barao, ${ }^{28}$ L. Barrin, ${ }^{15}$ A. Bartoloni, ${ }^{42}$ S. Başeğmez-du Pree, ${ }^{18, *}$ R. Battiston, ${ }^{39,40}$ U. Becker, ${ }^{10}$ M. Behlmann, ${ }^{10}$ B. Beischer, ${ }^{1}$ J. Berdugo, ${ }^{30}$ B. Bertucci, ${ }^{36,37}$ V. Bindi, ${ }^{20}$ W. de Boer, ${ }^{25}$ K. Bollweg, ${ }^{21}$ B. Borgia, ${ }^{42,43}$ M. J. Boschini, ${ }^{32}$ M. Bourquin, ${ }^{16}$ E. F. Bueno, ${ }^{18}$ J. Burger, ${ }^{10}$ W. J. Burger, ${ }^{39}$ S. Burmeister, ${ }^{26}$ X. D. Cai, ${ }^{10}$ M. Capell, ${ }^{10}$ J. Casaus,${ }^{30}$ G. Castellini, ${ }^{14}$ F. Cervelli, ${ }^{38}$ Y. H. Chang, ${ }^{47,48}$ G. M. Chen, ${ }^{6,7}$ H. S. Chen, ${ }^{6,7}$ Y. Chen,${ }^{16}$ L. Cheng, ${ }^{22}$ H. Y. Chou, ${ }^{48}$ S. Chouridou, ${ }^{1}$ V. Choutko, ${ }^{10}$ C. H. Chung, ${ }^{1}$ C. Clark, ${ }^{10,21}$ G. Coignet, ${ }^{3}$ C. Consolandi, ${ }^{20}$ A. Contin, ${ }^{8,9}$ C. Corti,${ }^{20}$ Z. Cui,${ }^{22,23}$ K. Dadzie, ${ }^{10}$ Y. M. Dai, ${ }^{5}$ C. Delgado, ${ }^{30}$ S. Della Torre ${ }^{32}$ M. B. Demirköz, ${ }^{2}$ L. Derome, ${ }^{17}$ S. Di Falco ${ }^{38}$ V. Di Felice, ${ }^{44, \dagger}$ C. Díaz, ${ }^{30}$ F. Dimiccoli,${ }^{39}$

P. von Doetinchem,${ }^{20}$ F. Dong, ${ }^{34}$ F. Donnini, ${ }^{44, \dagger}$ M. Duranti, ${ }^{36}$ A. Egorov,${ }^{10}$ A. Eline, ${ }^{10}$ J. Feng, ${ }^{10}$ E. Fiandrini, ${ }^{36,37}$ P. Fisher,${ }^{10}$ V. Formato, ${ }^{44, \dagger}$ C. Freeman, ${ }^{20}$ Y. Galaktionov, ${ }^{10}$ C. Gámez ${ }^{30}$ R. J. García-López, ${ }^{27}$ C. Gargiulo, ${ }^{15}$ H. Gast,${ }^{1}$ I. Gebauer, ${ }^{25}$ M. Gervasi, ${ }^{32,33}$ F. Giovacchini, ${ }^{30}$ D. M. Gómez-Coral, ${ }^{20}$ J. Gong, ${ }^{34}$ C. Goy, ${ }^{3}$ V. Grabski, ${ }^{31}$ D. Grandi, ${ }^{32,33}$ M. Graziani, ${ }^{36,37}$ K. H. Guo, ${ }^{19}$ S. Haino, ${ }^{47}$ K. C. Han,${ }^{29}$ R. K. Hashmani, ${ }^{2}$ Z. H. He, ${ }^{19}$ B. Heber,${ }^{26}$ T. H. Hsieh, ${ }^{10}$ J. Y. Hu, ${ }^{6,7}$ Z. C. Huang, ${ }^{19}$ M. Incagli, ${ }^{38}$ W. Y. Jang, ${ }^{13}$ Yi Jia,${ }^{10}$ H. Jinchi, ${ }^{29}$ K. Kanishev, ${ }^{39}$ B. Khiali, ${ }^{44 \dagger}$ G. N. Kim, ${ }^{13}$ Th. Kirn, ${ }^{1}$ M. Konyushikhin, ${ }^{10}$ O. Kounina, ${ }^{10}$ A. Kounine, ${ }^{10}$ V. Koutsenko, ${ }^{10}$ A. Kuhlman, ${ }^{20}$ A. Kulemzin, ${ }^{10}$ G. La Vacca, ${ }^{32,33}$

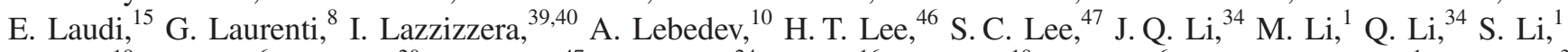
T. X. Li, ${ }^{19}$ Z. H. Li, ${ }^{6}$ C. Light, ${ }^{20}$ C. H. Lin, ${ }^{47}$ T. Lippert, ${ }^{24}$ Z. Liu, ${ }^{16}$ S. Q. Lu, ${ }^{19}$ Y. S. Lu, ${ }^{6}$ K. Luebelsmeyer, ${ }^{1}$ J. Z. Luo, ${ }^{34}$ S. S. Lyu, ${ }^{19}$ F. Machate, ${ }^{1}$ C. Mañá, ${ }^{30}$ J. Marín, ${ }^{30}$ J. Marquardt, ${ }^{26}$ T. Martin, ${ }^{10,21}$ G. Martínez, ${ }^{30}$ N. Masi,${ }^{8,9}$ D. Maurin, ${ }^{17}$ A. Menchaca-Rocha, ${ }^{31}$ Q. Meng, ${ }^{34}$ D. C. Mo, ${ }^{19}$ M. Molero, ${ }^{30}$ P. Mott, ${ }^{10,21}$ L. Mussolin, ${ }^{36,37}$ J. Q. Ni, ${ }^{19}$ N. Nikonov, ${ }^{1}$ F. Nozzoli ${ }^{39}$ A. Oliva, ${ }^{8}$ M. Orcinha, ${ }^{28}$ M. Palermo, ${ }^{20}$ F. Palmonari, ${ }^{8,9}$ M. Paniccia,${ }^{16}$ A. Pashnin, ${ }^{10}$ M. Pauluzzi,${ }^{36,37}$ S. Pensotti, ${ }^{32,33}$ H. D. Phan, ${ }^{10}$ R. Piandani, ${ }^{25}$ V. Plyaskin, ${ }^{10}$ S. Poluianov, ${ }^{35}$ X. M. Qi,${ }^{19}$ X. Qin, ${ }^{10}$ Z. Y. Qu,${ }^{47}$ L. Quadrani, ${ }^{8,9}$ P. G. Rancoita, ${ }^{32}$ D. Rapin, ${ }^{16}$ A. Reina Conde, ${ }^{27}$ S. Rosier-Lees, ${ }^{3}$ A. Rozhkov, ${ }^{10}$ D. Rozza,${ }^{32,33}$ R. Sagdeev, ${ }^{11}$ S. Schael, ${ }^{1}$ S. M. Schmidt, ${ }^{24}$ A. Schulz von Dratzig, ${ }^{1}$ G. Schwering, ${ }^{1}$ E. S. Seo, ${ }^{12}$ B. S. Shan, ${ }^{4}$ J. Y. Shi, ${ }^{34}$ T. Siedenburg, ${ }^{1}$ C. Solano, ${ }^{10}$

R. Sonnabend, ${ }^{1}$ J. W. Song, ${ }^{23}$ Q. Sun, ${ }^{23}$ Z. T. Sun,${ }^{6,7}$ M. Tacconi,${ }^{32,33}$ X. W. Tang, ${ }^{6}$ Z. C. Tang, ${ }^{6}$ J. Tian, ${ }^{36,37}$

Samuel C. C. Ting $\odot,{ }^{10,15}$ S. M. Ting, ${ }^{10}$ N. Tomassetti, ${ }^{36,37}$ J. Torsti, ${ }^{49}$ C. Tüysüz, ${ }^{2}$ T. Urban, ${ }^{10,21}$ I. Usoskin,${ }^{35}$ V. Vagelli,,${ }^{41, *}$ R. Vainio, ${ }^{49}$ E. Valente, ${ }^{42,43}$ E. Valtonen, ${ }^{49}$ M. Vázquez Acosta, ${ }^{27}$ M. Vecchi, ${ }^{18}$ M. Velasco, ${ }^{30}$ J. P. Vialle, ${ }^{3}$ C. Wallmann ${ }^{26}$ L. Q. Wang, ${ }^{23}$ N. H. Wang, ${ }^{23}$ Q. L. Wang, ${ }^{5}$ S. Wang, ${ }^{20}$ X. Wang, ${ }^{10}$ Z. X. Wang, ${ }^{19}$ J. Wei, ${ }^{16}$ Z. L. Weng, ${ }^{10}$ H. Wu, ${ }^{34}$ R. Q. Xiong, ${ }^{34}$ W. Xu, ${ }^{22,23}$ Q. Yan, ${ }^{10}$ Y. Yang, ${ }^{45}$ H. Yi, ${ }^{34}$ Y. J. Yu, Z. Q. Yu, ${ }^{6}$ M. Zannoni, ${ }^{32,33}$ C. Zhang, ${ }^{6}$ F. Zhang, ${ }^{6}$ F. Z. Zhang, ${ }^{6,7}$ J. H. Zhang, ${ }^{34}$ Z. Zhang, ${ }^{10}$ F. Zhao, ${ }^{6,7}$ Z. M. Zheng, ${ }^{4}$ H. L. Zhuang, ${ }^{6}$ V. Zhukov, ${ }^{1}$ A. Zichichi, ${ }^{8,9}$ N. Zimmermann, ${ }^{1}$ and P. Zuccon ${ }^{39,40}$

(AMS Collaboration)

${ }^{1}$ I. Physics Institute and JARA-FAME, RWTH Aachen University, 52056 Aachen, Germany

${ }^{2}$ Department of Physics, Middle East Technical University (METU), 06800 Ankara, Turkey

${ }^{3}$ Univ. Grenoble Alpes, Univ. Savoie Mont Blanc, CNRS, LAPP-IN2P3, 74000 Annecy, France ${ }^{4}$ Beihang University (BUAA), Beijing 100191, China

${ }^{5}$ Institute of Electrical Engineering (IEE), Chinese Academy of Sciences, Beijing 100190, China

${ }^{6}$ Institute of High Energy Physics (IHEP), Chinese Academy of Sciences, Beijing 100049, China

${ }^{7}$ University of Chinese Academy of Sciences (UCAS), Beijing 100049, China

${ }^{8}$ INFN Sezione di Bologna, 40126 Bologna, Italy

${ }^{9}$ Università di Bologna, 40126 Bologna, Italy

${ }^{10}$ Massachusetts Institute of Technology (MIT), Cambridge, Massachusetts 02139, USA

${ }^{11}$ East-West Center for Space Science, University of Maryland, College Park, Maryland 20742, USA

${ }^{12}$ IPST, University of Maryland, College Park, Maryland 20742, USA

${ }^{13}$ CHEP, Kyungpook National University, 41566 Daegu, Korea

${ }^{14}$ CNR-IROE, 50125 Firenze, Italy

${ }^{15}$ European Organization for Nuclear Research (CERN), 1211 Geneva 23, Switzerland

${ }^{16}$ DPNC, Université de Genève, 1211 Genève 4, Switzerland

${ }^{17}$ Univ. Grenoble Alpes, CNRS, Grenoble INP, LPSC-IN2P3, 38000 Grenoble, France

${ }^{18}$ Kapteyn Astronomical Institute, University of Groningen, 9700 AV Groningen, Netherlands 


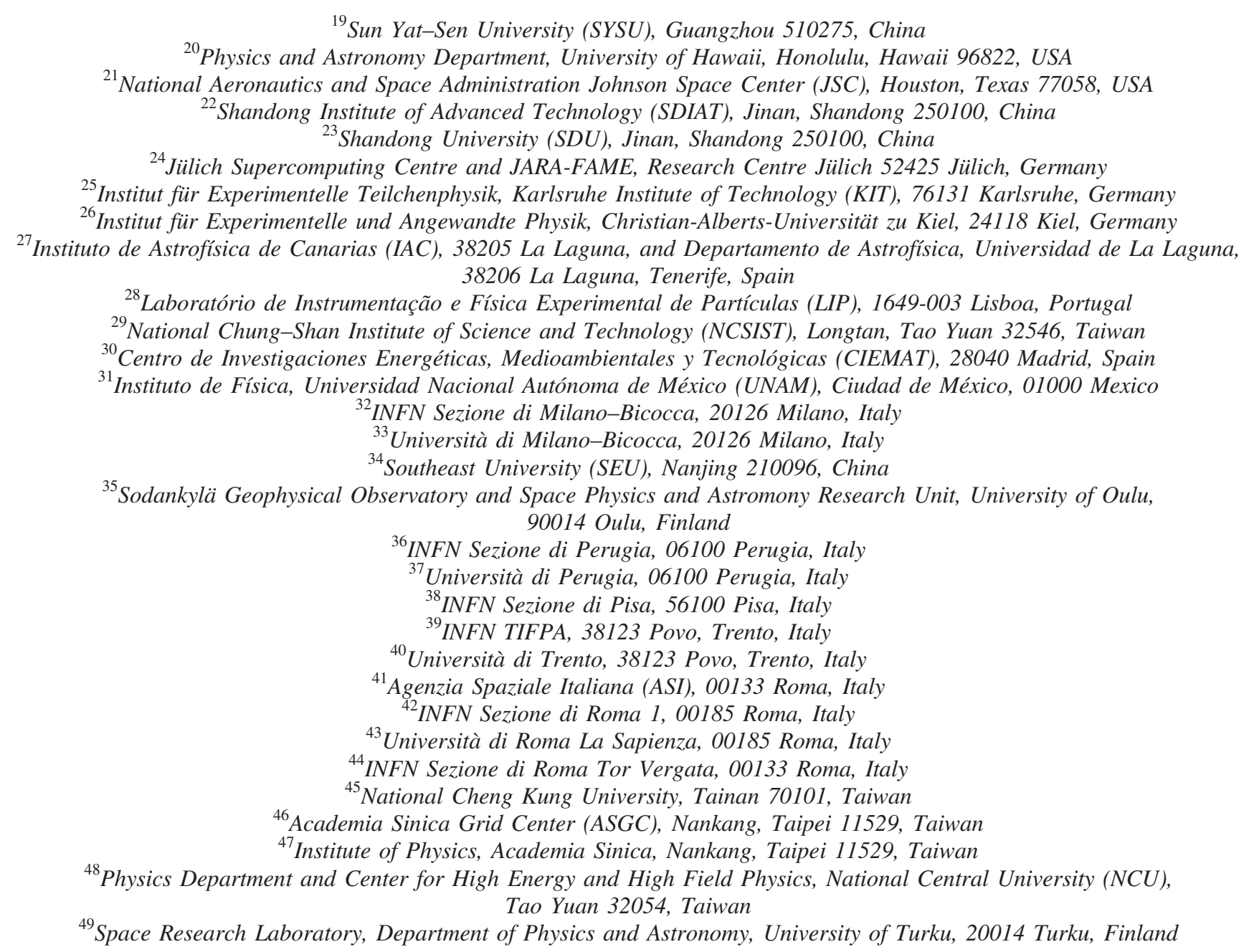

(Received 10 March 2020; accepted 20 April 2020; published 29 May 2020)

\begin{abstract}
We report the observation of new properties of primary cosmic rays, neon $(\mathrm{Ne})$, magnesium $(\mathrm{Mg})$, and silicon $(\mathrm{Si})$, measured in the rigidity range $2.15 \mathrm{GV}$ to $3.0 \mathrm{TV}$ with $1.8 \times 10^{6} \mathrm{Ne}, 2.2 \times 10^{6} \mathrm{Mg}$, and $1.6 \times 10^{6}$ Si nuclei collected by the Alpha Magnetic Spectrometer experiment on the International Space Station. The $\mathrm{Ne}$ and $\mathrm{Mg}$ spectra have identical rigidity dependence above $3.65 \mathrm{GV}$. The three spectra have identical rigidity dependence above $86.5 \mathrm{GV}$, deviate from a single power law above $200 \mathrm{GV}$, and harden in an identical way. Unexpectedly, above $86.5 \mathrm{GV}$ the rigidity dependence of primary cosmic rays $\mathrm{Ne}, \mathrm{Mg}$, and $\mathrm{Si}$ spectra is different from the rigidity dependence of primary cosmic rays $\mathrm{He}, \mathrm{C}$, and $\mathrm{O}$. This shows that the $\mathrm{Ne}, \mathrm{Mg}$, and $\mathrm{Si}$ and $\mathrm{He}, \mathrm{C}$, and $\mathrm{O}$ are two different classes of primary cosmic rays.
\end{abstract}

DOI: 10.1103/PhysRevLett.124.211102

Primary cosmic rays $\mathrm{Ne}, \mathrm{Mg}$, and $\mathrm{Si}$ are thought to be mainly produced and accelerated in astrophysical sources. Precise knowledge of their spectra in the gigavolt to teravolt rigidity region provides important information on the

Published by the American Physical Society under the terms of the Creative Commons Attribution 4.0 International license. Further distribution of this work must maintain attribution to the author(s) and the published article's title, journal citation, and DOI. origin, acceleration, and propagation processes of cosmic rays in the Galaxy [1]. Previously, the precision measurement of the primary cosmic rays $\mathrm{He}, \mathrm{C}$, and $\mathrm{O}$ fluxes with the Alpha Magnetic Spectrometer experiment (AMS) has been reported [2], revealing an identical rigidity dependence of these three fluxes above $60 \mathrm{GV}$, including the unexpected deviation from a single power law (hardening) of their spectra above $\sim 200 \mathrm{GV}$. Differences in the rigidity dependence of $\mathrm{Ne}, \mathrm{Mg}$, and $\mathrm{Si}$ compared to $\mathrm{He}, \mathrm{C}$, and $\mathrm{O}$ 
provide new insights into the origin and propagation of cosmic rays $[3,4]$.

Over the past 30 years there have been few measurements of $\mathrm{Ne}, \mathrm{Mg}$, and $\mathrm{Si}$ fluxes in kinetic energy per nucleon [5-11]. Typically these measurements have errors larger than $20 \%$ at $50 \mathrm{GeV} / \mathrm{n}$. There are no measurements of $\mathrm{Ne}, \mathrm{Mg}$, and $\mathrm{Si}$ fluxes in rigidity.

In this Letter we report the precise measurements of the $\mathrm{Ne}, \mathrm{Mg}$, and Si fluxes in the rigidity range from $2.15 \mathrm{GV}$ to $3.0 \mathrm{TV}$ based on $1.8 \times 10^{6} \mathrm{Ne}, 2.2 \times 10^{6} \mathrm{Mg}$, and $1.6 \times$ $10^{6} \mathrm{Si}$ nuclei collected by AMS during the first 7 years (May 19, 2011 to May 26, 2018) of operation aboard the International Space Station (ISS). The total error is 5\% at $100 \mathrm{GV}$ for each flux.

Detector.-The layout and description of the AMS detector are presented in Ref. [12]. The key elements used in this measurement are the permanent magnet [13], the nine layers $(L 1-L 9)$ of silicon tracker [14], and the four planes of time of flight (TOF) scintillation counters [15]. AMS also contains a transition radiation detector, a ring imaging Čerenkov detector, an electromagnetic calorimeter (ECAL), and an array of 16 anticoincidence counters. Together, the tracker and the magnet measure the rigidity $R$ of charged cosmic rays, with a maximum detectable rigidity of $3.2 \mathrm{TV}$ for $Z=10,3.1 \mathrm{TV}$ for $Z=12$, and $3.0 \mathrm{TV}$ for $Z=14$ over the $3 \mathrm{~m}$ lever arm. Further information on the layout and the performance of the detector is included in Refs. [16,17].

$\mathrm{Ne}, \mathrm{Mg}$, and $\mathrm{Si}$ traversing AMS were triggered as described in Ref. [18]. The trigger efficiencies have been measured to be $>94 \%$ for the three nuclei over the entire rigidity range.

Monte Carlo (MC) simulated events were produced using a dedicated program developed by the collaboration based on the GEANT4-10.1 package [19]. The program simulates electromagnetic and hadronic interactions of particles in the material of AMS and generates detector responses.

Event selection.-In the first 7 years AMS has collected $120 \times 10^{9}$ cosmic ray events. The collection time used in this analysis includes only those seconds during which the detector was in normal operating conditions and, in addition, AMS was pointing within $40^{\circ}$ of the local zenith and the ISS was outside of the South Atlantic Anomaly. Because of the geomagnetic field, this collection time increases with rigidity, reaching $1.74 \times 10^{8}$ seconds above $30 \mathrm{GV}$.

$\mathrm{Ne}, \mathrm{Mg}$, and $\mathrm{Si}$ events are required to be downward going and to have a reconstructed track in the inner tracker which passes through $L 1$. In the highest rigidity region, $R \geq 1.2 \mathrm{TV}$, the track is also required to pass through $L 9$. Track fitting quality criteria such as a $\chi^{2} /$ d.o.f. $<10$ in the bending coordinate are applied, similar to Refs. [18,20,21].

The measured rigidity is required to be greater than a factor of 1.2 times the maximum geomagnetic cutoff within the AMS field of view. The cutoff was calculated by backtracing [22] particles from the top of AMS out to 50 Earth's radii using the recent International Geomagnetic Reference Field model [23].

Charge measurements on $L 1$, the inner tracker, the upper TOF, the lower TOF, and, for $R>1.2 \mathrm{TV}, L 9$ are required to be compatible with charge $Z=10$ for Ne, $Z=12$ for $\mathrm{Mg}$, and $Z=14$ for Si. As an example, Fig. S1 of Supplemental Material (SM) [16] shows the charge measurement for the inner tracker alone. The charge selection yields purities of $>98 \%$ for $\mathrm{Ne}$ and $\mathrm{Mg}$ and $>99.7 \%$ for $\mathrm{Si}$. The impurities have two sources. The first source is a residual background from the interactions of heavy nuclei such as $\mathrm{Na}, \mathrm{Mg}, \mathrm{Al}$, and $\mathrm{Si}$ in the material between $L 1$ and $L 2$ (the transition radiation detector and upper TOF). It has been evaluated by fitting the charge distribution from $L 1$ with charge distribution templates of $\mathrm{Na}, \mathrm{Mg}, \mathrm{Al}$, and $\mathrm{Si}$ as shown in Fig. S2 of SM [16] for Ne. The charge distribution templates are obtained from a selection of noninteracting samples at $L 2$ by the use of the charge measurements with $L 1$, upper TOF, and $L 3-L 8$. This residual background is $<0.3 \%$ for the three nuclei over the entire rigidity range. The second source is a background from $\mathrm{Na}, \mathrm{Mg}, \mathrm{Al}, \mathrm{Si}$, $\mathrm{P}, \mathrm{S}$, and heavier nuclei interacting in materials above $L 1$ (thin support structures made of carbon fiber and aluminum honeycomb). It has been estimated from simulation using MC samples generated according to AMS flux measurements $[16,24]$ to be $<2 \%$ for $\mathrm{Ne},<1.5 \%$ for $\mathrm{Mg}$, and negligible for $\mathrm{Si}$ over the entire rigidity range.

After background subtraction we obtain $1.8 \times 10^{6}$ neon, $2.2 \times 10^{6}$ magnesium, and $1.6 \times 10^{6}$ silicon nuclei. The overall uncertainty due to background subtraction is $<0.5 \%$ for the three nuclei over the entire rigidity range.

Data analysis. - The isotropic flux $\Phi_{i}$ in the $i$ th rigidity bin $\left(R_{i}, R_{i}+\Delta R_{i}\right)$ is given by

$$
\Phi_{i}=\frac{N_{i}}{A_{i} \epsilon_{i} T_{i} \Delta R_{i}},
$$

where $N_{i}$ is the number of events corrected for bin-to-bin migration; $A_{i}$ is the effective acceptance including geometric acceptance, event reconstruction and selection efficiencies, and inelastic interactions of nuclei in the AMS materials, as described below; $\epsilon_{i}$ is the trigger efficiency; and $T_{i}$ is the collection time. In this Letter the fluxes were measured in 66 bins from $2.15 \mathrm{GV}$ to 3.0 TV, with bin widths chosen according to the rigidity resolution. The bin widths are identical for the three nuclei and are identical with our previous publication on $\mathrm{He}$, $\mathrm{C}$, and $\mathrm{O}[2]$ with the exception of the first bin and the last four bins.

The bin-to-bin migration of events was corrected using the unfolding procedure described in Ref. [20]. These corrections, $\left(N_{i}-\boldsymbol{\aleph}_{i}\right) / \boldsymbol{\aleph}_{i}$, where $\boldsymbol{\aleph}_{i}$ is the number of observed events in bin $i$, are $+18 \%$ at $3 \mathrm{GV},+9 \%$ at 
$5 \mathrm{GV},-2 \%$ at $150 \mathrm{GV}$, and $-5 \%$ at $3 \mathrm{TV}$ for $\mathrm{Ne}$ and very similar for $\mathrm{Mg}$ and $\mathrm{Si}$.

Extensive studies were made of the systematic errors. These errors include the uncertainties in the background evaluation discussed above, the trigger efficiency, the geomagnetic cutoff factor, the acceptance calculation, the rigidity resolution function, and the absolute rigidity scale.

The systematic error on the fluxes associated with the trigger efficiency measurement is $<1 \%$ for these nuclei over the entire rigidity range.

The geomagnetic cutoff factor was varied from 1.0 to 1.4, resulting in a negligible systematic uncertainty $(<0.1 \%)$ in the rigidity range below $30 \mathrm{GV}$.

The effective acceptances $A_{i}$ were calculated using MC simulation and corrected for small differences between the data and simulated events related to (a) event reconstruction and selection, namely in the efficiencies of velocity vector determination, track finding, charge determination, and tracker quality cuts, and (b) the details of inelastic interactions of nuclei in the AMS materials. The systematic errors on the fluxes associated with the reconstruction and selection are $<1 \%$ over the entire rigidity range for the three nuclei.

The material traversed by nuclei from the top of AMS to $L 9$ is composed primarily of carbon and aluminum. The survival probabilities for $\mathrm{Ne}, \mathrm{Mg}$, and $\mathrm{Si}$ nuclei due to interactions in the materials were measured using cosmic ray data collected by AMS as described in Ref. [25]. The systematic error due to uncertainties in evaluation of inelastic cross sections of all the materials traversed is $<3.5 \%$ up to $100 \mathrm{GV}$ for the three fluxes. Above $100 \mathrm{GV}$, the small rigidity dependence of the cross sections from the Glauber-Gribov model [19] was treated as an uncertainty and added in quadrature to the uncertainties from the measured interaction probabilities [25]. The corresponding systematic errors on the three fluxes in the range of $100 \mathrm{GV}$ to $3 \mathrm{TV}$ were evaluated to be $<4 \%$.

The rigidity resolution functions $\Delta(1 / R)$ for $\mathrm{Ne}, \mathrm{Mg}$, and $\mathrm{Si}$ have a pronounced Gaussian core characterized by widths $\sigma$ and non-Gaussian tails more than $2.5 \sigma$ away from the center [18]. The resolution functions have been verified with the procedures described in detail in Ref. [21]. As an example, Fig. S3 of SM [16] shows that the measured tracker bending coordinate accuracies are in a good agreement with the simulation. The systematic error on the fluxes due to the rigidity resolution functions was obtained by repeating the unfolding procedure while varying the widths of the Gaussian cores of the resolution functions by $5 \%$ and by independently varying the amplitudes of the non-Gaussian tails by $10 \%$. The resulting systematic error on the fluxes is less than $1 \%$ below $300 \mathrm{GV}$ and $2.5 \%$ at 3 TV for these nuclei.

There are two contributions to the systematic uncertainty on the rigidity scale [20]. The first is due to residual tracker misalignment. This error was estimated by comparing the
$E / p$ ratio for electrons and positrons, where $E$ is the energy measured with the electromagnetic calorimeter and $p$ is the momentum measured with the tracker. It was found to be $1 / 30 \mathrm{TV}^{-1}$ [26]. The second systematic error on the rigidity scale arises from the magnetic field map measurement and its temperature corrections. The error on the fluxes due to uncertainty on the rigidity scale is $<1 \%$ up to $300 \mathrm{GV}$ and $6 \%$ at $3 \mathrm{TV}$.

Most importantly, several independent analyses were performed on the same data sample by different study groups. The results of those analyses are completely consistent with this Letter.

Results.-The measured $\mathrm{Ne}, \mathrm{Mg}$, and $\mathrm{Si}$ fluxes including statistical and systematic errors are reported in Tables SISIII of SM [16] as functions of the rigidity at the top of the AMS detector. To examine the difference in rigidity dependences of the $\mathrm{Ne}, \mathrm{Mg}$, and $\mathrm{Si}$ fluxes, the $\mathrm{Ne} / \mathrm{Mg}$ and $\mathrm{Si} / \mathrm{Mg}$ flux ratios were computed using the data in Tables SI-SIII of SM [16] and reported in Tables SIV and SV of SM [16] with their statistical and systematic errors.

Figure 1(a) shows the $\mathrm{Ne}$ and $\mathrm{Mg}$ fluxes and $\mathrm{Ne} / \mathrm{Mg}$ flux ratio, and Fig. 1(b) shows the $\mathrm{Si}$ and $\mathrm{Mg}$ fluxes and $\mathrm{Si} / \mathrm{Mg}$ flux ratio, as functions of rigidity $\tilde{R}$ with the total errors, the sum in quadrature of statistical and systematic errors. In this and the subsequent figures, the points are placed along the abscissa at $\tilde{R}$ calculated for a flux $\propto R^{-2.7}$ [27]. To establish the rigidity intervals where the $\mathrm{Ne}, \mathrm{Mg}$, and $\mathrm{Si}$ fluxes have identical rigidity dependence, the fits of $\mathrm{Ne} / \mathrm{Mg}$ and $\mathrm{Si} / \mathrm{Mg}$ ratios have been performed to

$$
\frac{\Phi_{\mathrm{Ne}, \mathrm{Si}}}{\Phi_{\mathrm{Mg}}}= \begin{cases}k\left(R / R_{0}\right)^{\Delta} & R \leq R_{0} \\ k & R>R_{0} .\end{cases}
$$

For the $\mathrm{Ne} / \mathrm{Mg}$ ratio, the fit yields $k^{\mathrm{Ne} / \mathrm{Mg}}=$ $0.84 \pm 0.02, \quad R_{0}^{\mathrm{Ne} / \mathrm{Mg}}=3.65 \pm 0.5 \mathrm{GV}, \quad$ and $\quad \Delta^{\mathrm{Ne} / \mathrm{Mg}}=$ $0.19 \pm 0.08$ with $\chi^{2} /$ d.o.f. $=42 / 64$ over the entire rigidity range. From the fit results we found that the $\mathrm{Ne}$ and $\mathrm{Mg}$ fluxes have an identical rigidity dependence above 3.65 GV. Surprisingly, AMS has also observed an identical rigidity dependence above $7 \mathrm{GV}$ between secondary cosmic ray $\mathrm{Li}$ and $\mathrm{B}$ fluxes [28].

For the $\mathrm{Si} / \mathrm{Mg}$ ratio, the fit yields $k^{\mathrm{Si} / \mathrm{Mg}}=0.89 \pm 0.02$, $R_{0}^{\mathrm{Si} / \mathrm{Mg}}=86.5 \pm 13 \mathrm{GV}, \quad$ and $\quad \Delta^{\mathrm{Si} / \mathrm{Mg}}=0.069 \pm 0.005$ with $\chi^{2} /$ d.o.f. $=29 / 53$ above $6 \mathrm{GV}$.

From the fit results we conclude that all three fluxes have an identical rigidity dependence above $86.5 \mathrm{GV}$. This is a unique observation of the properties of $\mathrm{Ne}, \mathrm{Mg}$, and $\mathrm{Si}$ fluxes.

Figure 2(a) shows the neon, Fig. 2(b) the magnesium, and Fig. 2(c) the silicon fluxes as a function of kinetic energy per nucleon $E_{K}$ together with earlier measurements [5-11]. Data from other experiments have been extracted using Ref. [29]. 

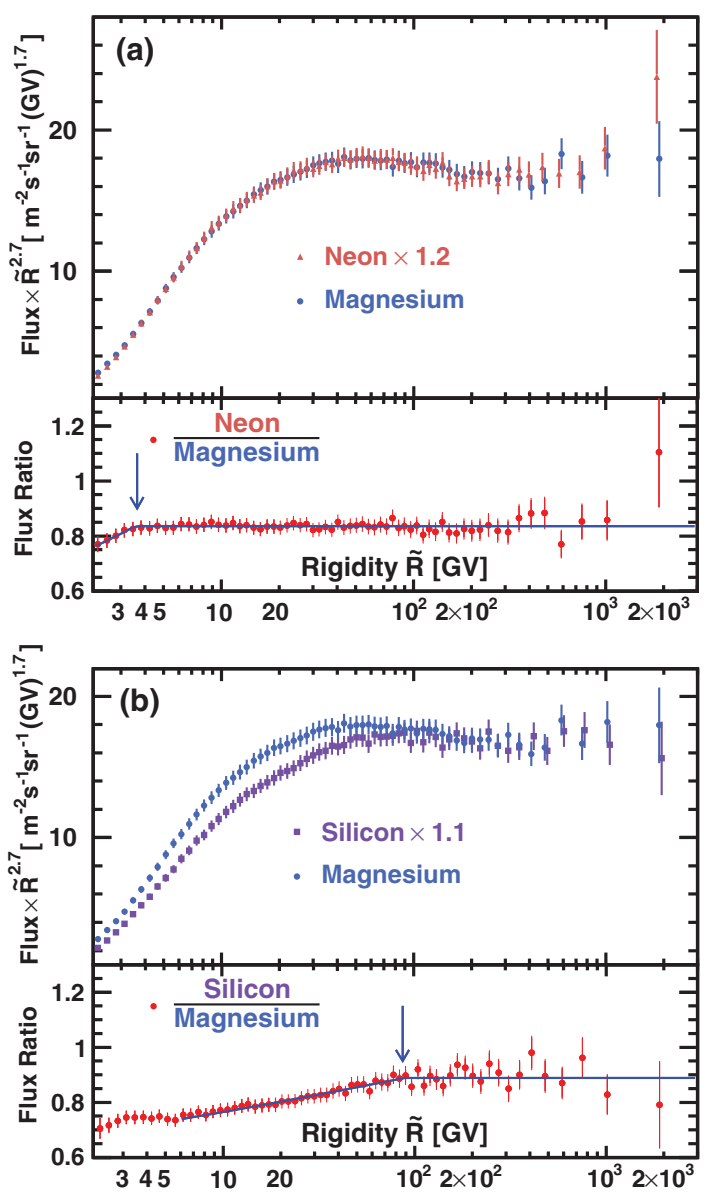

FIG. 1. (a) $\mathrm{Ne}$ and $\mathrm{Mg}$ fluxes multiplied by $\tilde{R}^{2.7}$ and $\mathrm{Ne} / \mathrm{Mg}$ flux ratio, and (b) $\mathrm{Si}$ and $\mathrm{Mg}$ fluxes multiplied by $\tilde{R}^{2.7}$ and $\mathrm{Si} / \mathrm{Mg}$ flux ratio with their total errors as functions of rigidity. For display purposes only, the $\mathrm{Ne}$ and $\mathrm{Si}$ fluxes were rescaled as indicated. For clarity, $\mathrm{Ne}$ and $\mathrm{Si}$ data points above $400 \mathrm{GV}$ are displaced horizontally. The solid curves show the fit results with Eq. (2). As seen, the $\mathrm{Ne}$ and $\mathrm{Mg}$ fluxes have identical rigidity dependence above $3.65 \mathrm{GV}$ and the three fluxes have identical rigidity dependence above $86.5 \mathrm{GV}$, as indicated by the location of the arrows.

To examine the rigidity dependence of the fluxes, the variation of the flux spectral indices with rigidity was obtained in a model independent way from

$$
\gamma=d[\log (\Phi)] / d[\log (R)]
$$

over nonoverlapping rigidity intervals bounded by 7.09 , 12.0, 16.6, 28.8, 45.1, 86.5, 192.0, 441.0, and 3000.0 GV. The results are presented in Fig. 3. As seen, the $\mathrm{Ne}$ and $\mathrm{Mg}$ spectral indices are identical in this rigidity range and the three flux spectral indices harden identically with rigidity above $\sim 200 \mathrm{GV}$.

To compare the rigidity dependence of the $\mathrm{Ne}, \mathrm{Mg}$, and $\mathrm{Si}$ fluxes with that of $\mathrm{He}, \mathrm{C}$, and $\mathrm{O}$ primary cosmic ray fluxes, which have identical rigidity dependence above $60 \mathrm{GV}$ [2], the ratios of the neon, magnesium, and silicon

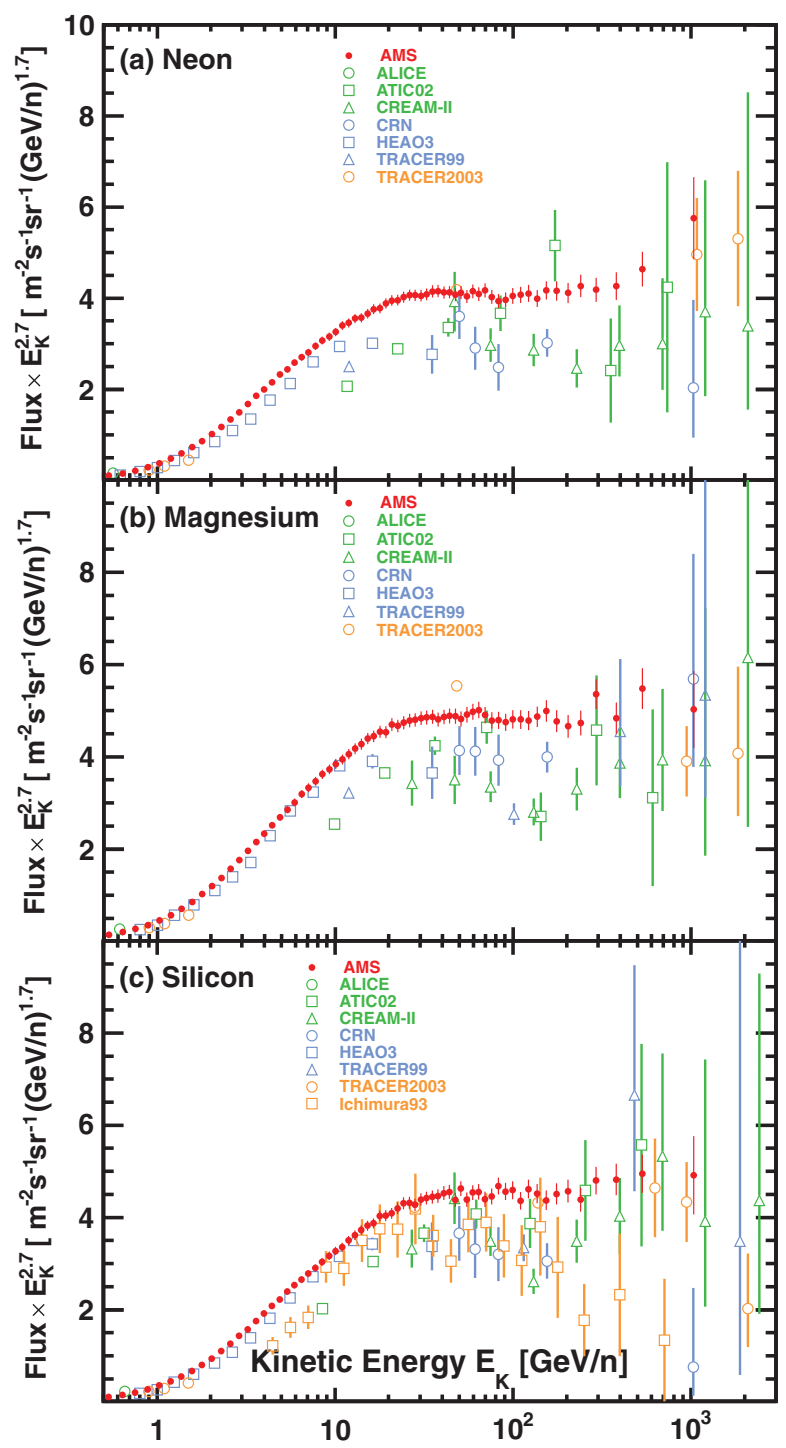

FIG. 2. The AMS (a) neon, (b) magnesium, and (c) silicon fluxes as functions of kinetic energy per nucleon $E_{K}$ multiplied by $E_{K}^{2.7}$ together with earlier measurements. For the AMS measurement $E_{K}=\left(\sqrt{Z^{2} \tilde{R}^{2}+M^{2}}-M\right) / A$, where $Z, M$, and $A$ are ${ }_{10}^{20} \mathrm{Ne},{ }_{12}^{24} \mathrm{Mg}$, and ${ }_{14}^{28} \mathrm{Si}$ nuclei charge, mass, and atomic mass numbers, respectively.

fluxes to the oxygen flux were computed using the data in Tables SI-SIII of SM [16] and data in Ref. [30], and reported in Tables SVI-SVIII of SM [16], with statistical and systematic errors. To examine the rigidity dependence of $\mathrm{Ne} / \mathrm{O}, \mathrm{Mg} / \mathrm{O}$, and $\mathrm{Si} / \mathrm{O}$ flux ratios, fits to the double power law,

$$
\frac{\Phi_{\mathrm{Ne}, \mathrm{Mg}, \mathrm{Si}}}{\Phi_{\mathrm{O}}}= \begin{cases}C(R / 86.5 \mathrm{GV})^{\Delta} & R \leq 86.5 \mathrm{GV} \\ C(R / 86.5 \mathrm{GV})^{\delta} & R>86.5 \mathrm{GV}\end{cases}
$$

where $C$ is a constant, to the ratios for rigidities above $20 \mathrm{GV}$ have been performed and shown in Fig. S4 of 


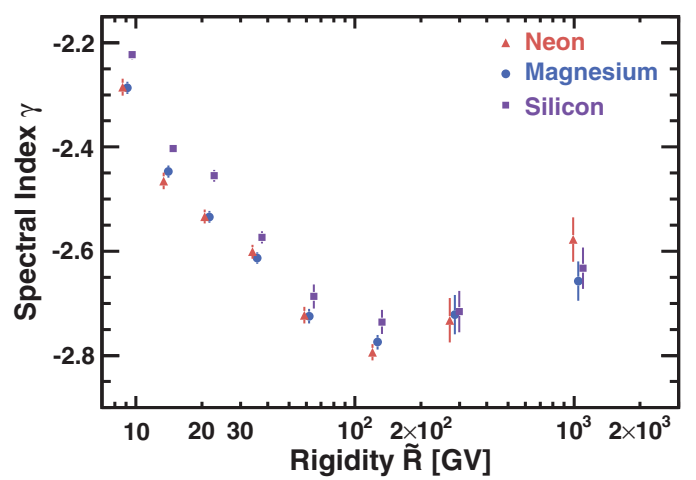

FIG. 3. The dependence of the $\mathrm{Ne}, \mathrm{Mg}$, and $\mathrm{Si}$ spectral indices on rigidity. For clarity, the $\mathrm{Ne}$ and $\mathrm{Si}$ data points are displaced horizontally. As seen, the $\mathrm{Ne}$ and $\mathrm{Mg}$ spectral indices are identical in this rigidity range and the three flux spectral indices harden identically with rigidity above $\sim 200 \mathrm{GV}$.

SM [16]. Figure 4 shows the rigidity dependence of the spectral indices $\mathrm{Ne} / \mathrm{O}, \mathrm{Mg} / \mathrm{O}$, and $\mathrm{Si} / \mathrm{O}$ obtained from the fits. As seen, above $86.5 \mathrm{GV}$ the spectral indices are $\delta^{\mathrm{Ne} / \mathrm{O}}=-0.046 \pm 0.010, \quad \delta^{\mathrm{Mg} / \mathrm{O}}=-0.049 \pm 0.011$, and $\delta^{\mathrm{Si} / \mathrm{O}}=-0.040 \pm 0.011$, fully compatible with each other and incompatible with zero. Their average value is $\langle\delta\rangle=-0.045 \pm 0.008$. The difference of $\langle\delta\rangle$ from zero by more than $5 \sigma$ shows that the $\mathrm{Ne}, \mathrm{Mg}$, and $\mathrm{Si}$ is a different class of primary cosmic rays than $\mathrm{He}, \mathrm{C}$, and $\mathrm{O}$.

This is illustrated in Fig. 5, which shows the rigidity dependence of the $\mathrm{Ne}, \mathrm{Mg}$, and $\mathrm{Si}$ fluxes compared to rigidity dependence of the $\mathrm{He}, \mathrm{C}$, and $\mathrm{O}$ fluxes from Ref. [30] above $86.5 \mathrm{GV}$ together with the fit results of $\mathrm{He}$, $\mathrm{C}$, and $\mathrm{O}$ fluxes and $\mathrm{Ne}, \mathrm{Mg}$, and $\mathrm{Si}$ fluxes with a function

$$
\Phi=C\left(\frac{R}{45 \mathrm{GV}}\right)^{\gamma}\left[1+\left(\frac{R}{R_{0}}\right)^{\Delta \gamma / s}\right]^{s}
$$

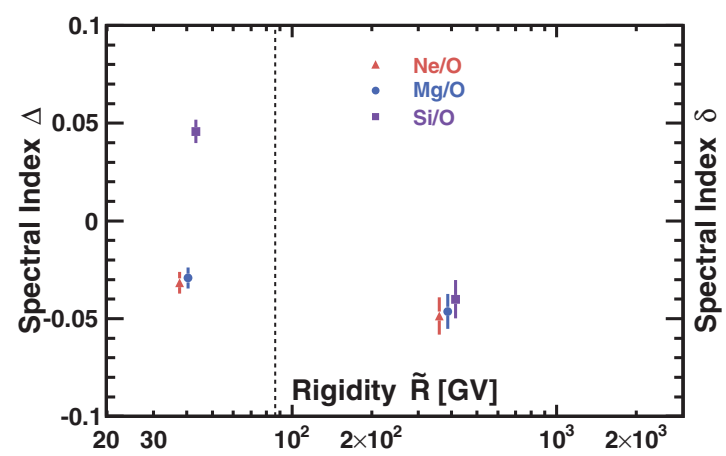

FIG. 4. The AMS Ne/O, $\mathrm{Mg} / \mathrm{O}$, and $\mathrm{Si} / \mathrm{O}$ flux ratio spectral indices obtained with fits of Eq. (4) as a function of rigidity. For clarity, $\mathrm{Ne} / \mathrm{O}$ and $\mathrm{Si} / \mathrm{O}$ spectral indices data points are displaced horizontally. The vertical dashed line shows the interval boundary of $86.5 \mathrm{GV}$. As seen, above $86.5 \mathrm{GV}$ all spectral indices are identical with average value $\langle\delta\rangle=-0.045 \pm 0.008$. where $C$ is the normalization constant and $s$ quantifies the smoothness of the transition of the spectral index from $\gamma$ for rigidities below the characteristic transition rigidity $R_{0}$ to $\gamma+\Delta \gamma$ for rigidities above $R_{0}$ [18]. The details of the fit procedures and parameters obtained are provided in the SM [16].

As seen, the rigidity dependences of $\mathrm{Ne}, \mathrm{Mg}$, and $\mathrm{Si}$ and $\mathrm{He}, \mathrm{C}$, and $\mathrm{O}$ are distinctly different.

The previous AMS results on primary cosmic rays $\mathrm{He}$, $\mathrm{C}$, and $\mathrm{O}[2]$ show, unexpectedly, that they have identical rigidity dependence above $60 \mathrm{GV}$ and that they deviate from a single power law above $200 \mathrm{GV}$, whereas the secondary cosmic rays $\mathrm{Li}, \mathrm{Be}$, and $\mathrm{B}$ also have identical rigidity dependence above $30 \mathrm{GV}$ and deviate from a single power law above $200 \mathrm{GV}$. The rigidity dependence of primary cosmic rays $\mathrm{He}, \mathrm{C}$, and $\mathrm{O}$ is distinctly different from secondary cosmic rays $\mathrm{Li}, \mathrm{Be}$, and B [28]. These results indicate there are two kinds of cosmic ray rigidity dependences. These observations have generated new developments in cosmic ray models [4,31]. The theoretical models have their limitations, as none of them predicted the observed spectral behavior of the cosmic rays. The results in this Letter on heavier primary cosmic rays $\mathrm{Ne}, \mathrm{Mg}$, and $\mathrm{Si}$ show that primary cosmic rays have at least two distinct classes of rigidity dependence. These unexpected results together with ongoing measurements of heavier elements in cosmic rays will enable us to determine how many classes of rigidity dependence exist in both primary and secondary cosmic rays and provide important input to the development of the theoretical models.

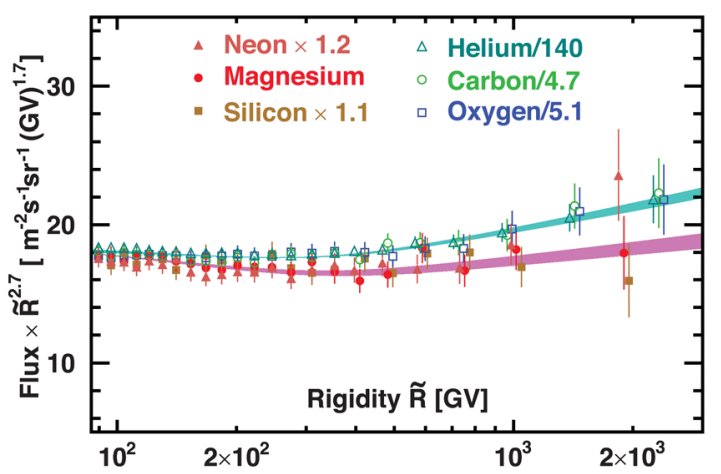

FIG. 5. The rigidity dependence of the $\mathrm{Ne}, \mathrm{Mg}$, and $\mathrm{Si}$ fluxes compared to rigidity dependence of the $\mathrm{He}, \mathrm{C}$, and $\mathrm{O}$ fluxes from Ref. [30] above 86.5 GV. For display purposes only, the He, C, O, $\mathrm{Ne}$, and $\mathrm{Si}$ fluxes were rescaled as indicated. For clarity, He, O, $\mathrm{Ne}$, and Si data points above $400 \mathrm{GV}$ are displaced horizontally. The green shaded area shows the fit result of $\mathrm{He}, \mathrm{C}$, and $\mathrm{O}$ fluxes from Ref. [30] with Eq. (5) together with fit errors [16]. The magenta shaded area shows the fit result of $\mathrm{Ne}, \mathrm{Mg}$, and $\mathrm{Si}$ fluxes from Ref. [16] with Eq. (5) when varying $\gamma_{\mathrm{NeMgSi}}=\gamma_{\mathrm{HeCO}}+\langle\delta\rangle$, by \pm 0.008 , from the value of $\langle\delta\rangle=-0.045 \pm 0.008$. 
In conclusion, we have presented precision measurements of the $\mathrm{Ne}, \mathrm{Mg}$, and $\mathrm{Si}$ fluxes rigidity dependence from $2.15 \mathrm{GV}$ to $3.0 \mathrm{TV}$, with detailed studies of the systematic errors. The $\mathrm{Ne}$ and $\mathrm{Mg}$ spectra have identical rigidity dependence above $3.65 \mathrm{GV}$. The three spectra have identical rigidity dependence above $86.5 \mathrm{GV}$, deviate from a single power law above $200 \mathrm{GV}$, and harden in an identical way. Unexpectedly, above $86.5 \mathrm{GV}$ the rigidity dependence of $\mathrm{Ne}, \mathrm{Mg}$, and $\mathrm{Si}$ spectra is different from the rigidity dependence of primary cosmic rays $\mathrm{He}, \mathrm{C}$, and $\mathrm{O}$, which have identical rigidity dependence above $60 \mathrm{GV}$ and harden above $200 \mathrm{GV}$. Above $86.5 \mathrm{GV}$, the $\mathrm{Ne} / \mathrm{O}, \mathrm{Mg} / \mathrm{O}$, and $\mathrm{Si} / \mathrm{O}$ ratios can be described by a simple power law $\propto R^{\delta}$ with $\langle\delta\rangle=-0.045 \pm 0.008$. This shows that the $\mathrm{Ne}$, $\mathrm{Mg}$, and $\mathrm{Si}$ and $\mathrm{He}, \mathrm{C}$, and $\mathrm{O}$ are two different classes of primary cosmic rays. These are new and unexpected properties of primary cosmic rays.

We are grateful for important physics discussions with Pasquale Blasi, Fiorenza Donato, Jonathan Ellis, Jonathan Feng, Mischa Malkov, Igor Moskalenko (in particular for illuminating discussions on the important papers in Ref. [4]), and Subir Sarkar. We thank former NASA Administrator Daniel S. Goldin for his dedication to the legacy of the ISS as a scientific laboratory and his decision for NASA to fly AMS as a DOE payload. We also acknowledge the continuous support of the NASA leadership, particularly William H. Gerstenmaier, and of the JSC and MSFC flight control teams that have allowed AMS to operate optimally on the ISS for over eight years. We are grateful for the support of Jim Siegrist and his staff of the DOE including resources from the National Energy Research Scientific Computing Center under Contract No. DE-AC02-05CH11231. We also acknowledge the continuous support from MIT and its School of Science, Michael Sipser and Boleslaw Wyslouch. Research supported by CAS, NSFC, MOST, the provincial governments of Shandong, Jiangsu, Guangdong, and the China Scholarship Council, China; the Academy of Finland, Project No. 321882, Finland; CNRS/IN2P3 and CNES, France; Pascale Ehrenfreund, DLR under Grants No. 50001403 and No. 50001805 and JARA-HPC under Project No. JARA0052, Germany; INFN and ASI under ASI-INFN Agreements No. 2019-19-HH.0 and No. 2014037-R.0, and ASI-University of Perugia Agreement No. 2019-2-HH.0, Italy; CHEP and NRF under Grant No. NRF-2018R1A6A1A06024970 at Kyungpook National University, Korea; the Consejo Nacional de Ciencia y Tecnología and UNAM, Mexico; NWO under Grant. No. 680-1-004, Netherlands; FCT under Grant No. CERN/FIS-PAR/0013/2019, Portugal; CIEMAT, IAC, CDTI, and SEIDI-MINECO under Grants No. ESP2017-87055-C2-1-P, No. SEV-2015-0548, No. MDM-2015-0509, and No. RyC-2013-14660, Spain; the Swiss National Science Foundation (SNSF), federal and cantonal authorities, and the Fondation Dr. Manfred
Steuer, Switzerland; Academia Sinica and the Ministry of Science and Technology (MOST) under Grants No. 103-2112-M-006-018-MY3, No. 105-2112-M-001003, and No. CDA-105-M06, former Presidents of Academia Sinica Yuan-Tseh Lee and Chi-Huey Wong and former Ministers of MOST Maw-Kuen Wu and Luo-Chuan Lee, Taiwan; the Turkish Atomic Energy Authority under Grants No. 2017TAEK(CERN) A5.H6.F2-15, Turkey; and NSF Grants No. 14255202 and No. 1551980, Wyle Laboratories Grant No. 2014/ T72497, and NASA NESSF Grant No. HELIO15F-0005, U.S. We gratefully acknowledge the strong support from CERN including Fabiola Gianotti, and the CERN IT department including Bernd Panzer-Steindel, and from the European Space Agency including Johann-Dietrich Wörner and Simonetta Di Pippo.

*Also at: Nikhef, 1098 XG Amsterdam, Netherlands.

${ }^{\dagger}$ Also at: ASI Space Science Data Center (SSDC), 00133 Roma, Italy.

${ }^{\star}$ Also at: INFN Sezione di Perugia, 06100 Perugia, Italy.

[1] I. A. Grenier, J. H. Black, and A. W. Strong, Annu. Rev. Astron. Astrophys. 53, 199 (2015); P. Blasi, Astron. Astrophys. Rev. 21, 70 (2013); A. W. Strong, I. V. Moskalenko, and V.S. Ptuskin, Annu. Rev. Nucl. Part. Sci. 57, 285 (2007); A. Castellina and F. Donato, Astropart. Phys. 24, 146 (2005).

[2] M. Aguilar et al., Phys. Rev. Lett. 119, 251101 (2017).

[3] G. Jóhannesson et al., Astrophys. J. 824, 16 (2016).

[4] M. J. Boschini et al., Astrophys. J. 840, 115 (2017); 858, 61 (2018). In the 2018 paper, for each individual primary cosmic ray $\mathrm{He}, \mathrm{C}$, and $\mathrm{O}$, a set of distinct parameters are chosen so that, with their model, they can describe our measured data.

[5] K. A. Lave et al., Astrophys. J. 770, 117 (2013); J. A. Esposito et al., Astropart. Phys. 1, 33 (1992); M. Ichimura et al. Phys. Rev. D 48, 1949 (1993).

[6] J. J. Engelmann et al., Astron. Astrophys. 233, 96 (1990).

[7] S. P. Swordy, D. Müller, P. Meyer, J. L'Heureux, and J. M. Grunsfeld, Astrophys. J. 349, 625 (1990).

[8] A. D. Panov et al., Bull. Russ. Acad. Sci. 73, 564 (2009).

[9] H. S. Ahn et al., Astrophys. J. 715, 1400 (2010); 714, L89 (2010); 707, 593 (2009).

[10] F. Gahbauer, G. Hermann, J. R. Hörandel, D. Müller, and A. A. Radu, Astrophys. J. 607, 333 (2004).

[11] M. Ave, P. J. Boyle, F. Gahbauer, C. Höppner, J. R. Hörandel, M. Ichimura, D. Müller, and A. Romero-Wolf, Astrophys. J. 678, 262 (2008).

[12] A. Kounine, Int. J. Mod. Phys. E 21, 1230005 (2012); S. Rosier-Lees, in Proceedings of Astroparticle Physics TEVPA/IDM, Amsterdam, 2014 (unpublished); S. Ting, Nucl. Phys. B, Proc. Suppl. 243-244, 12 (2013); S.-C. Lee, in Proceedings of the 20th International Conference on Supersymmetry and Unification of Fundamental Interactions (SUSY 2012), Beijing, 2012 (unpublished); M. Aguilar, in Proceedings of the XL International Meeting on Fundamental Physics, Centro de Ciencias de Benasque 
Pedro Pascual, 2012 (unpublished); S. Schael, in Proceedings of the 10th Symposium on Sources and Detection of Dark Matter and Dark Energy in the Universe, Los Angeles, 2012 (unpublished); B. Bertucci, Proc. Sci. EPS-HEP2011 (2011) 67; M. Incagli, AIP Conf. Proc. 1223, 43 (2010); R. Battiston, Nucl. Instrum. Methods Phys. Res., Sect. A 588, 227 (2008).

[13] K. Lübelsmeyer et al., Nucl. Instrum. Methods Phys. Res., Sect. A 654, 639 (2011).

[14] B. Alpat et al., Nucl. Instrum. Methods Phys. Res., Sect. A 613, 207 (2010).

[15] V. Bindi et al., Nucl. Instrum. Methods Phys. Res., Sect. A 743, 22 (2014).

[16] See Supplemental material at http://link.aps.org/ supplemental/10.1103/PhysRevLett.124.211102 for details of the AMS detector; for details of the fits of the $\mathrm{He}, \mathrm{C}$, and $\mathrm{O}$ and $\mathrm{Ne}, \mathrm{Mg}$, and Si fluxes with Eq. (5); for the tabulated neon, magnesium, and silicon fluxes, neon and silicon to magnesium flux ratios, neon, magnesium, and silicon to oxygen flux ratios, all as functions of rigidity; and figures regarding charge resolution and selection, tracker coordinate resolution, and $\mathrm{Ne}, \mathrm{Mg}$, and $\mathrm{Si}$ to $\mathrm{O}$ flux ratios versus rigidity.

[17] G. Ambrosi, V. Choutko, C. Delgado, A. Oliva, Q. Yan, and Y. Li, Nucl. Instrum. Methods Phys. Res., Sect. A 869, 29 (2017).

[18] M. Aguilar et al., Phys. Rev. Lett. 115, 211101 (2015).

[19] J. Allison et al., Nucl. Instrum. Methods Phys. Res., Sect. A 835, 186 (2016); IEEE Trans. Nucl. Sci. 53, 270 (2006);
S. Agostinelli et al., Nucl. Instrum. Methods Phys. Res., Sect. A 506, 250 (2003).

[20] M. Aguilar et al., Phys. Rev. Lett. 114, 171103 (2015).

[21] M. Aguilar et al., Phys. Rev. Lett. 117, 231102 (2016).

[22] J. Alcaraz et al., Phys. Lett. B 484, 10 (2000).

[23] C. C. Finlay et al., Geophys. J. Int. 183, 1216 (2010); E. Thébault et al., Earth Planets Space 67, 79 (2015).

[24] M. Aguilar et al. (AMS Collaboration), Measurement of the heavy nuclei cosmic rays fluxes with the Alpha Magnetic Spectrometer on the International Space Station (to be published).

[25] Q. Yan, V. Choutko, A. Oliva, and M. Paniccia, Nucl. Phys. A996, 121712 (2020).

[26] J. Berdugo, V. Choutko, C. Delgado, and Q. Yan, Nucl. Instrum. Methods Phys. Res., Sect. A 869, 10 (2017).

[27] G. D. Lafferty and T. R. Wyatt, Nucl. Instrum. Methods Phys. Res., Sect. A 355, 541 (1995). We have used Eq. (6) with $\tilde{R} \equiv x_{l w}$.

[28] M. Aguilar et al., Phys. Rev. Lett. 120, 021101 (2018).

[29] D. Maurin, F. Melot, and R. Taillet, Astron. Astrophys. 569, A32 (2014).

[30] M. Aguilar et al., The Alpha Magnetic Spectrometer (AMS) on the International Space Station: Part II-Results from the first seven years (to be published).

[31] C. Evoli, R. Aloisio, and P. Blasi, Phys. Rev. D 99, 103023 (2019); M. J. Boschini et al., Astrophys. J. 889, 167 (2020); N. Weinrich, Y. Génolini, M. Boudaud, L. Derome, and D. Maurin, Astron. Astrophys., https://doi.org/10.1051/ 0004-6361/202037875. 